\title{
Frailty is a useful predictive marker of postoperative complications after pancreaticoduodenectomy
}

\author{
Yutaka Nakano 1*0, Yuki Hirata', Tatsuya Shimogawara', Toru Yamada', Koki Mihara', Ryo Nishiyama', \\ Shin Nishiya ${ }^{1}$, Hideki Taniguchi ${ }^{2}$ and Tomohisa Egawa'
}

\begin{abstract}
Background: Frailty results in a high risk for disability, hospitalization, and mortality. This study aimed to investigate perioperative details of frail patients who underwent pancreatectomy and whether frailty can be a predictive factor of postoperative complications, especially of clinically relevant postoperative pancreatic fistula (CR-POPF).

Methods: This retrospective study included patients who underwent pancreatectomy in our hospital between August 2016 and March 2019. The patients were divided into frail and pre-/non-frail groups. The diagnostic criteria were based on the Japanese version of the Cardiovascular Health Study.

Results: Of 93 patients, $11(11.8 \%)$ and 82 (88.2\%) were frail and pre-/non-frail patients, with median ages of 82 and 72 years, respectively ( $p=0.041$ ). Postoperative complications (Clavien-Dindo $\geqq I I l a)$ were found in 8 and 32 patients $(p=0.034)$, CR-POPF in 3 and 13 patients $(p=0.346)$, and postoperative hospital stays were 21 and 17 days $(p=0.041)$, respectively. On multivariate analysis, frailty was an independent predictive factor (odds ratio [OR] 5.604, 95.0\% confidence interval [CI] 1.002-30.734; $p=0.047$ ) of postoperative complications (Clavien-Dindo $\geqq I I l a)$ after pancreaticoduodenectomy. On multivariate analysis, a soft pancreas (OR 5.696, 95.0\% Cl 1.142-28.149; $p=0.034$ ) was an independent and significant predictive factor of CR-POPF after pancreaticoduodenectomy.
\end{abstract}

Conclusions: Frailty may be a useful predictive factor of postoperative complications in patients undergoing pancreaticoduodenectomy.

Keywords: Frailty, Sarcopenia, Pancreaticoduodenectomy, Postoperative complications, Clinically relevant postoperative pancreatic fistula

\section{Background}

Frailty has become the center of attention in the geriatric field because it is considered to result in a high risk for falls, disability, hospitalization, and mortality [1]. Pancreatectomy remains one of the most life-threatening abdominal surgeries associated with mortality [2]. The

\footnotetext{
*Correspondence: yutaka.nakano.9833@gmail.com

${ }^{1}$ Department of Surgery, Saiseikai Yokohamashi Tobu Hospital, 3-6-1 Shimosueyoshi, Tsurumi-ku, Yokohama, Kanagawa 230-0012, Japan Full list of author information is available at the end of the article
}

proportion of the elderly population has increased not only in other countries but also in Japan [3], which in turn has increased the number of elderly patients undergoing pancreatectomy. Many pancreatectomies have been performed for malignancy, and compared with younger patients, elderly patients are at a risk for increased morbidity and mortality [4]. The risk of frailty is higher in the elderly population, and frailty predicts severe complications and mortality after pancreatectomies [5]. The safety of pancreatectomy performed in community cancer centers is

C C The Author(s). 2020 Open Access This article is licensed under a Creative Commons Attribution 4.0 International License, which permits use, sharing, adaptation, distribution and reproduction in any medium or format, as long as you give appropriate credit to the original author(s) and the source, provide a link to the Creative Commons licence, and indicate if changes were made. The images or other third party material in this article are included in the article's Creative Commons licence, unless indicated otherwise in a credit line to the material. If material is not included in the article's Creative Commons licence and your intended use is not permitted by statutory regulation or exceeds the permitted use, you will need to obtain permission directly from the copyright holder. To view a copy of this licence, visit http://creativecommons.org/licenses/by/4.0/ The Creative Commons Public Domain Dedication waiver (http://creativecommons.org/publicdomain/zero/1.0/) applies to the data made available in this article, unless otherwise stated in a credit line to the data. 
similar to that performed in any academic center or university hospital [6]. However, accurate evaluation and reduction of preoperative risk in the elderly population are essential, especially among community cancer centers.

Sarcopenia can be considered one of the main physical drivers of frailty or even a precursor state [7], and it has been considered one of the risk stratification tools to better identify potentially high-risk surgical patients [8]. A systematic review and meta-analysis [9] has reported an increase in the duration of inpatient hospital stay of sarcopenia patients. Several reports $[5,10,11]$ have reported frailty as an important independent predictor of outcomes after pancreatic surgery; however, to the best of our knowledge, the relationship between frail patients and pre-/non-frail patients or that between frailty and sarcopenia has not been extensively studied.

Thus, in this study, our primary aim was to evaluate the relationship between frailty and sarcopenia and investigate the clinicopathological characteristics of frail patients who had pancreatic resection, focusing on perioperative short-term outcomes, such as postoperative complications, especially postoperative pancreatic fistula (POPF). Moreover, our secondary aim was to evaluate whether frailty can be a predictive factor of postoperative complications (Clavien-Dindo classification $\geq$ IIIa) (CD $\geq$ IIIa) or clinically relevant postoperative pancreatic fistula (CR-POPF; grades B/C POPF).

\section{Methods}

\section{Patients}

Data of patients who underwent intended curative pancreatectomy (distal pancreatectomy and pancreaticoduodenectomy) at our institution between August 2016 and March 2019, were retrospectively reviewed. We excluded patients who were made to change surgical procedure to total pancreatectomy. This retrospective observational study used the "opt-out" method of our hospital. The study was approved by the Ethics Committee of Saiseikai Yokohamashi Tobu Hospital (ethical approval number: 20190032). Research was conducted in accordance with the Declaration of Helsinki 1975.

\section{Preoperative assessment in patient support center}

Since 2016, our hospital has established a patient support center where various conditions of preoperative patients have been assessed by anesthesiologists, nurses, pharmacists, registered dietitians, and dental hygienists from the viewpoint of enhanced recovery after surgery program [12]. In the center, demographic and clinical variables such as age, sex, body mass index, presence or absence of smoking (current and former) and alcohol intake history, past medical history, and medicines used (especially antithrombotic drugs) were assessed. Moreover, preoperative laboratory data (serum albumin, lymphocyte, total cholesterol, and hemoglobin levels, prognostic nutritional index [13], and controlling nutritional status score [14]) were evaluated.

\section{Definition of sarcopenia and frailty}

In the patient support center, we asked patients regarding their health condition, such as weight loss, physical activity, and walking speed, and measured grip strength. Multifrequency bioelectrical impedance analysis (InBody 770; Biospace, Tokyo, Japan) was performed to assess preoperative skeletal muscle mass. In this study, we defined sarcopenia according to the criteria of the Asian Working Group for Sarcopenia [15], and to diagnose frailty, we used the Japanese version of the cardiovascular health study (J-CHS) criteria, which was similar to previous studies that used the CHS criteria to identify frailty [16]. The J-CHS criteria comprise five items and questions-(i) shrinking: have you lost $\geq 2 \mathrm{~kg}$ in the past 6 months?; (ii) low activity: do you engage in moderate levels of physical exercise or sports aimed at health?; and do you engage in low levels of physical exercise aimed at health?; (iii) exhaustion: in the past 2 weeks, have you felt tired without a reason? (iv) weakness: grip strength $<26 \mathrm{~kg}$ in men or 18 $\mathrm{kg}$ in women; and (v) slowness: gait speed $<1.0 \mathrm{~m} / \mathrm{s}$. Frailty, pre-frailty, and non-frailty are defined as having $3-$ $5,1-2$, and 0 components, respectively. To investigate the relationship between frailty and sarcopenia, we adopted the J-CHS criteria, which included similar items to the criteria of sarcopenia, such as grip strength and walking time as weakness and slowness, respectively.

\section{Surgery and postoperative assessment}

Surgery included pancreaticoduodenectomy or distal pancreatectomy for malignant and benign tumors. D2 lymph node dissection was performed in all cancer patients. To evaluate the pancreas intraoperatively as soft or hard, surgeons judged the pancreas status subjectively (Fig. 1). Postoperative complications (e.g., POPF, bile leakage, fluid collection, intra-abdominal bleeding, and delayed gastric emptying) were evaluated according to the Clavien-Dindo classification. In this study, we especially focused on CRPOPF according to 2016 the International Study Group of Pancreatic Fistula classification [17].

\section{Statistical analyses}

Patients were divided into the frail group and the pre-/ non-frail group based on their frailty status defined according to the J-CHS criteria. The clinicopathological characteristics between the frail group and the pre-/nonfrail group and between the frail group and the sarcopenia group were evaluated. Categorical variables were compared using the chi-square test or Fisher's exact test, and continuous variables were compared using the Mann-Whitney $U$ test. Variables that were significant in 

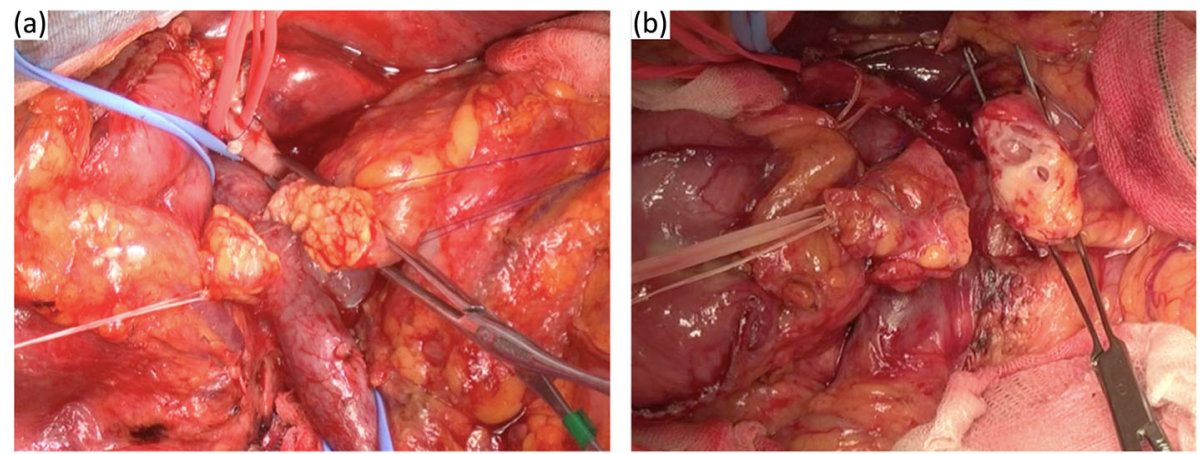

Fig. 1 Cut margin of the soft pancreas (a) and hard pancreas (b) during pancreaticoduodenectomy

the univariate analysis $(p<0.10)$ were included in the logistic regression analyses to identify independent predictive factors of postoperative complications $(\mathrm{CD} \geq$ IIIa) and CR-POPF. We analyzed independent predictive factors of not only postoperative complications $(\mathrm{CD} \geq$ IIIa) but also CR-POPF in the pancreaticoduodenectomy group and distal pancreatectomy group separately because the proposed mechanism of pancreatic fistula is different between pancreaticoduodenectomy and distal pancreatectomy [18].

All statistical analyses were performed using Statistical Package for the Social Sciences for Macintosh, software version 25.0 (IBM Corp., Armonk, NY, USA). $P<0.05$ was considered statistically significant.

\section{Results}

Patient characteristics in the frail, pre-/non-frail, frail, and sarcopenia groups

In total, 95 patients underwent curative pancreatectomy between August 2016 and March 2019. Of them, two patients had undergone total pancreatectomy and one patient had schizophrenia; as we could not perform accurate evaluation at the patient support center, they were excluded. Therefore, 93 patients were enrolled for the analysis. Of the 93 patients, $11(11.8 \%)$ patients were included in the frail group and 82 (88.2\%) patients were included in the pre-/non-frail group. The overall patient characteristics and demographic and clinical characteristics of the frail and pre-/non-frail group are listed in Table 1. All frail patients had sarcopenia; hence, we compared frail patients with sarcopenic patients who did not satisfy the J-CHS criteria in terms of their clinical characteristics, including details of postoperative complications with $\mathrm{CD}$ $\geq$ IIIa (Table 2). The clinical characteristic details of the 11 frail patients are shown in Table 3.

\section{Predictive factors for postoperative complications (CD $\geq$ IIla) and CR-POPF after pancreaticoduodenectomy and distal pancreatectomy}

Predictive factors associated with postoperative complications (CD $\geq$ IIIa) and CR-POPF in the pancreaticoduodenectomy $(N=68,73.1 \%)$ and distal pancreatectomy $(N=25,26.9 \%)$ groups are shown in Tables 4 and 5. In multivariate analysis, frailty (odds ratio [OR] 5.604, 95.0\% confidence interval $[\mathrm{CI}] 1.022-30.734 ; p=$ 0.047 ) was the only independent and significant predictive factor of postoperative complications (CD $\geq$ IIIa) in the pancreaticoduodenectomy group. In contrast, soft pancreas (OR 5.696, 95.0\% CI 1.142-28.149; $p=0.034$ ) an independent and significant predictive factor of CR-POPF in the pancreaticoduodenectomy group. In this study, both univariate and multivariate analyses did not reveal predictive factors of postoperative complications (CD $\geq$ IIIa) and CR-POPF in the distal pancreatectomy group.

\section{Discussion}

This study investigated not only clinical characteristics between frail and pre-/non-frail patients and frail patients with sarcopenic patients but also predictive factors related to postoperative complications and CR-POPF. In this study, frailty and soft pancreas were an independent and significant predictive factor of postoperative complications (CD $\geq \mathrm{IIIa}$ ) and CR-POPF after pancreaticoduodenectomy, respectively.

Many physicians often observe that some patients can withstand operational stress, while others cannot despite being of the same chronological age, and they judge instinctively and subjectively whether patients have the physiological reserve to endure operations and postoperative burdens. Although some older patients do not have such reserve to endure surgical stress [19], there are appropriate methods for evaluating older surgical patients. Our results demonstrate that frailty may be a useful predictive factor of postoperative complications in patients undergoing pancreatectomy and may become one of the risk stratification tools to better identify potentially highrisk surgical patients. Unlike sarcopenia, frailty represents not only the skeletal muscle mass and muscle function but also physical activity in daily living, weight loss, and social isolation [6]. Thus, frailty is considered a biologic syndrome of decreased reserve and resistance to 
Table 1 Demographic and clinical characteristics or all patients and between frail and pre-/non-frail patients

\begin{tabular}{|c|c|c|c|c|}
\hline & Total $(N=93)$ & Frail $(N=11)$ & Pre-/non-frail $(N=82)$ & $p$ value \\
\hline Age (years) & $72(27-88)$ & $82(69-88)$ & $72(27-85)$ & 0.041 \\
\hline Sex (male/female) & $57 / 36$ & $4 / 7$ & $53 / 29$ & 0.071 \\
\hline Body mass index $\left(\mathrm{kg} / \mathrm{m}^{2}\right)$ & $21.7(14.2-33.0)$ & $19.7(14.5-24.9)$ & $22.0(14.2-33.0)$ & 0.242 \\
\hline Smoking (current and former) & 45 (48.4\%) & $2(18.2 \%)$ & $43(52.4 \%)$ & 0.033 \\
\hline Alcohol & $6(6.5 \%)$ & $0(0.0 \%)$ & $6(7.1 \%)$ & 0.350 \\
\hline Diabetes mellitus & $23(25.9 \%)$ & $5(45.5 \%)$ & $18(22.0 \%)$ & 0.090 \\
\hline Antithrombotic drugs & $21(22.6 \%)$ & $5(45.5 \%)$ & $16(19.5 \%)$ & 0.053 \\
\hline Grip strength (kg) & $27.0(10.0-48.0)$ & $14.6(10.0-22.8)$ & $27.4(13.1-48.0)$ & 0.002 \\
\hline Skeletal muscle index $\left(\mathrm{kg} / \mathrm{m}^{2}\right)$ & $6.7(4.0-8.9)$ & $4.7(4.0-5.7)$ & $6.8(4.7-8.9)$ & 0.063 \\
\hline Sarcopenia & 37 (39.8\%) & $11(100.0 \%)$ & $26(31.7 \%)$ & $<0.001$ \\
\hline Disease & & & & 0.539 \\
\hline Pancreatic cancer & $46(49.5 \%)$ & $6(54.5 \%)$ & $40(48.8 \%)$ & \\
\hline Bile duct cancer (including papilla of Vater) & $20(21.5 \%)$ & $4(36.4 \%)$ & $16(19.5 \%)$ & \\
\hline Intraductal papillary mucinous neoplasm & $9(9.7 \%)$ & $0(0.0 \%)$ & $9(11.0 \%)$ & \\
\hline Pancreatic neuroendocrine tumor & $6(6.5 \%)$ & $0(0.0 \%)$ & $6(7.3 \%)$ & \\
\hline Benign tumor & $5(5.4 \%)$ & $0(0.0 \%)$ & $5(6.1 \%)$ & \\
\hline Others & $7(7.5 \%)$ & $1(9.1 \%)$ & $6(7.3 \%)$ & \\
\hline Surgical procedure & & & & 0.488 \\
\hline Pancreatoduodenectomy & $68(73.1 \%)$ & $9(81.8 \%)$ & $59(72.0 \%)$ & \\
\hline Distal pancreatectomy & $25(26.9 \%)$ & $2(18.2 \%)$ & $23(28.0 \%)$ & \\
\hline Soft pancreas & $62(66.7 \%)$ & $7(63.6 \%)$ & $55(67.1 \%)$ & 0.820 \\
\hline Albumin $(g / l)$ & $4.0(2.6-4.9)$ & $3.3(2.6-4.0)$ & $4.0(2.7-4.9)$ & 0.009 \\
\hline Lymphocyte $\left(\times 10^{3} / \mu \mathrm{l}\right)$ & $1551(530-3724)$ & $1242(530-2124)$ & $1568(540-3724)$ & 0.546 \\
\hline Total cholesterol (mg/dl) & $198(72-335)$ & $137(72-230)$ & $200(89-335)$ & 0.156 \\
\hline Hemoglobin (g/dl) & $12.9(8.4-19.6)$ & $11.3(9.4-14.0)$ & $13.1(8.4-19.6)$ & 0.059 \\
\hline Prognostic nutritional index & $48.2(32.1-62.6)$ & $36.2(32.3-48.2)$ & $49.0(32.1-62.6)$ & 0.002 \\
\hline Controlling nutritional status & & & & $<0.001$ \\
\hline 01 or 24 & $84(90.3 \%)$ & $4(36.4 \%)$ & $80(97.6 \%)$ & \\
\hline 58 or 8 & $9(9.7 \%)$ & $7(63.6 \%)$ & $2(2.4 \%)$ & \\
\hline Operative time (min) & $514(206-874)$ & $563(228-874)$ & $503(206-872)$ & 0.162 \\
\hline Blood loss (g) & $685(75-5671)$ & $985(223-2703)$ & $662(75-5671)$ & 0.186 \\
\hline Intraoperative transfusion & $20(21.5 \%)$ & $6(54.5 \%)$ & $14(17.1 \%)$ & 0.005 \\
\hline Clavien-Dindo classification $\geqq I I l a$ & $40(43.0 \%)$ & $8(72.7 \%)$ & $32(39.0 \%)$ & 0.034 \\
\hline Clinically relevant postoperative pancreatic fistula & $16(17.2 \%)$ & $3(27.3 \%)$ & $13(15.9 \%)$ & 0.346 \\
\hline Postoperative hospital stay (day) & $18(7-431)$ & $21(14-83)$ & $17(7-431)$ & 0.041 \\
\hline Postoperative 30-day mortality & $1(1.1 \%)$ & $1(9.1 \%)$ & $0(0.0 \%)$ & 0.006 \\
\hline Postoperative 90-day mortality & $3(3.2 \%)$ & $3(27.3 \%)$ & $0(0.0 \%)$ & $<0.001$ \\
\hline
\end{tabular}

Values in median

stressors, resulting from cumulative decline across multiple physiologic systems and causing vulnerability to adverse outcomes [1]. Our findings suggest that frailty is a more effective predictor than sarcopenia to evaluate potentially high-risk surgical patients, even if these two conditions start to converge because of their close relationship with the aging process [6].
Several reports [9-11] have revealed that frailty is an important predictor of postoperative morbidity and mortality after pancreatectomy, which is consistent with our study results. These studies used the modified frailty index (mFI) to define frailty [20], while our study used the J-CHS criteria. The mFI is a simple frailty assessment tool mainly evaluated by the patient's historical 
Table 2 Demographic and clinical characteristics between frail and sarcopenia

\begin{tabular}{|c|c|c|c|}
\hline & \multicolumn{2}{|l|}{ Sarcopenia $(N=37)$} & \multirow{2}{*}{$\begin{array}{l}p \\
\text { value }\end{array}$} \\
\hline & Frail $(N=11,29.7 \%)$ & Not frail $^{*}(N=26,70.3 \%)$ & \\
\hline Age (years) & $82(69-88)$ & $76(59-85)$ & 0.026 \\
\hline Sex (male/female) & $4 / 7$ & $13 / 13$ & 0.447 \\
\hline \multicolumn{4}{|l|}{ Medical history } \\
\hline Diabetes mellitus & $5(45.5 \%)$ & $4(15.4 \%)$ & 0.051 \\
\hline Cardiac valvular disease & $2(18.2 \%)$ & $0(0.0 \%)$ & 0.025 \\
\hline Myocardial infarction & $2(18.2 \%)$ & $2(7.7 \%)$ & 0.348 \\
\hline Chronic pulmonary disease or pneumonia & $4(36.4 \%)$ & $0(0.0 \%)$ & 0.001 \\
\hline Hypertension requiring medication & $3(27.3 \%)$ & 7 (26.9\%) & 0.546 \\
\hline Cerebrovascular accident & $3(27.3 \%)$ & $0(0.0 \%)$ & 0.004 \\
\hline Albumin $(g / l)$ & $3.3(2.6-4.0)$ & $3.7(3.1-4.6)$ & 0.218 \\
\hline Prognostic nutritional index & $36.2(32.3-48.2)$ & $47.1(38.8-58.9)$ & 0.540 \\
\hline Controlling nutritional status & & & $<0.001$ \\
\hline 01 or 24 & $4(36.4 \%)$ & $26(100 \%)$ & \\
\hline 58 or 8 & $7(63.6 \%)$ & $0(0.0 \%)$ & \\
\hline Operative time (min) & $563(228-874)$ & $497(206-753)$ & 0.122 \\
\hline Blood loss (g) & $985(223-2703)$ & $646(75-2613)$ & 0.122 \\
\hline Intraoperative transfusion & $6(54.5 \%)$ & $5(19.2 \%)$ & 0.032 \\
\hline Clavien-Dindo classification $\geqq I I I a$ & $8(72.7 \%)$ & $10(38.5 \%)$ & 0.057 \\
\hline Clinically relevant postoperative pancreatic fistula & $3(27.3 \%)$ & 7 (26.9\%) & 0.983 \\
\hline Intra-abdominal abscess & $1(9.1 \%)$ & $2(7.7 \%)$ & 0.887 \\
\hline Bile leakage & $0(0.0 \%)$ & $1(3.8 \%)$ & 0.510 \\
\hline Wound dehiscence & $1(9.1 \%)$ & $1(3.8 \%)$ & 0.519 \\
\hline Organ/space surgical site infection & $1(9.1 \%)$ & $0(0.0 \%)$ & 0.119 \\
\hline Respiratory failure & $3(27.3 \%)$ & $0(0.0 \%)$ & 0.005 \\
\hline Postoperative hospital stay (day) & $21(14-83)$ & $18(8-431)$ & 0.408 \\
\hline Postoperative 30-day mortality & $1(9.1 \%)$ & $0(0.0 \%)$ & 0.119 \\
\hline Postoperative 90-day mortality & $3(27.3 \%)$ & $0(0.0 \%)$ & 0.005 \\
\hline
\end{tabular}

Values in median

*Not frail: sarcopenia patients who were not satisfied the J-CHS criteria

variables, such as history of myocardial infarction, previous coronary operation, chronic obstructive pulmonary disease, or pneumonia. In contrast, the J-CHS criteria are mainly comprise patients' physical ability and conditions, such as shrinking, weakness, poor endurance, slowness, and low activity. Although it is important to focus on a patient's historical variables, such as $\mathrm{mFI}$, we aimed to investigate the relationship between frailty and sarcopenia, which is a progressive and generalized skeletal muscle disorder involving the accelerated loss of muscle mass and function. Thus, we adopted the J-CHS criteria, which included similar items to the criteria of sarcopenia, such as grip strength and walking time. Unlike these previous studies, our study focused on the relationship between frailty and sarcopenia. Table 2 shows that compared with sarcopenia patients who did not satisfied the J-CHS criteria, frail patients had pulmonary, neurologic, or cardiac medical histories and diabetes mellitus, which may influence postoperative morbidity and mortality after pancreatectomy. Moreover, frail patients had more postoperative complications with $\mathrm{CD} \geq$ IIIa than sarcopenia (not frail) patients $(p=0.087)$. No difference in the occurrence frequency of CR-POPF was found between the two groups, but a significant difference was found in the occurrence frequency of respiratory failure $(p=0.030)$, which resulted in postoperative mortality in frail patients. Sarcopenia was a risk stratification tool to better identify potentially high-risk surgical patients [7], but frailty was also a useful predictive factor of postoperative complications and may be an effective risk stratification tool to identify these potentially high-risk surgical patients.

Our report also focused on CR-POPF, which was not discussed in previous reports [9-11]. CR-POPF remains 
Table 3 Details of the clinical characteristics of frail patients

\begin{tabular}{|c|c|c|c|c|c|c|c|c|}
\hline No & $\begin{array}{l}\text { Age } \\
\text { (years) }\end{array}$ & Sex & $\begin{array}{l}\text { Medical } \\
\text { history }\end{array}$ & Disease & $\begin{array}{l}\text { Surgical } \\
\text { procedure }\end{array}$ & $\begin{array}{l}\text { Postoperative complications (Clavien- } \\
\text { Dindo classification } \geqq I I I a)\end{array}$ & $\begin{array}{l}\text { 30-day postoperative } \\
\text { mortality (cause of } \\
\text { death) }\end{array}$ & $\begin{array}{l}\text { 90-day postoperative } \\
\text { mortality (cause of } \\
\text { death) }\end{array}$ \\
\hline 1 & 86 & $\mathrm{~F}$ & $\begin{array}{l}\text { AS, heart } \\
\text { pacemaker }\end{array}$ & $P C$ & $\mathrm{DP}$ & Pancreatic fistula (grade B) & No & No \\
\hline 2 & 88 & $\mathrm{~F}$ & AS, DM & PC & PD & & No & No \\
\hline 3 & 88 & $\mathrm{~F}$ & $\mathrm{Cl}, \mathrm{DM}, \mathrm{PE}$ & $\mathrm{BC}$ & PD & $\begin{array}{l}\text { Pancreatic fistula (grade C), } \\
\text { pseudoaneurysm s/o, pylethrombosis, } \\
\text { melena }\end{array}$ & No & $\begin{array}{l}\text { Yes (acute respiratory } \\
\text { failure) }\end{array}$ \\
\hline 4 & 81 & M & Bronchiectasis & $P C$ & PD & $\begin{array}{l}\text { Bacterial pneumonia, ARDS, wound } \\
\text { dehiscence }\end{array}$ & No & $\begin{array}{l}\text { Yes (acute respiratory } \\
\text { failure, DIC) }\end{array}$ \\
\hline 5 & 72 & M & Gastric caner & $\begin{array}{l}\text { Remnant } \\
\text { gastric } \\
\text { cancer }\end{array}$ & PD & $\begin{array}{l}\text { Esophagojejunostomy leakage, } \\
\text { pancreatic fistula (grade C), aspiration } \\
\text { pneumonia }\end{array}$ & $\begin{array}{l}\text { Yes (acute respiratory } \\
\text { failure, septic shock) }\end{array}$ & No \\
\hline 6 & 69 & M & $\mathrm{Cl}, \mathrm{Ml}$ & $\mathrm{BC}$ & PD & Intra-abdominal abscess & No & No \\
\hline 7 & 82 & M & $\mathrm{Cl}, \mathrm{MI}, \mathrm{PAD}$ & $B C$ & PD & Paralytic ileus & No & No \\
\hline 8 & 79 & $\mathrm{~F}$ & Gastric ulcer & $B C$ & PD & $\begin{array}{l}\text { Anastomotic bleeding of } \\
\text { gastrojejunostomy }\end{array}$ & No & No \\
\hline 9 & 85 & $\mathrm{~F}$ & $\begin{array}{l}\text { Pulmonary } \\
\text { tuberculosis }\end{array}$ & PC & PD & & No & No \\
\hline 10 & 87 & $\mathrm{~F}$ & $\mathrm{DM}$ & PC & DP & Organ/space surgical site infection & No & No \\
\hline 11 & 81 & $\mathrm{~F}$ & $\begin{array}{l}\text { Duodenal } \\
\text { ulcer }\end{array}$ & PC & PD & & No & No \\
\hline
\end{tabular}

Abbreviations: $A R D S$ acute respiratory distress syndrome, $A S$ aortic stenosis, $B C$ bill duct cancer, $C l$ cerebral infarction; $D I C$ disseminated intravascular coagulation, $D M$ diabetes mellitus, $D P$ distal pancreatectomy, $F$ female, $M$ male, $M I$ myocardial infarction, $P A D$ peripheral arterial disease, $P C$ pancreatic cancer, $P D$ pancreaticoduodenectomy, $P E$ pulmonary embolism

one of the most life-threatening postoperative complications, and two frail patients in our study, who died within 90 days after pancreaticoduodenectomy, had CRPOPF. The direct cause of death of these patients was acute respiratory failure, which could have triggered uncontrollable CR-POPF. Frail patients may not have physiological reserve to endure postoperative lifethreatening complications, such as CR-POPF. Several reports $[21,22]$ considered that the soft texture of the pancreatic parenchyma could contribute to the development of POPF after pancreaticoduodenectomy. A soft pancreas and a small-diameter pancreatic duct preserve exocrine function, which increases the secretion of pancreatic juice and pressure within the pancreaticoenteric lumen [22]; our findings were consistent with these findings. However, in our study, "soft" pancreas was subjectively judged by the surgeons. Moreover, "soft" or "hard" pancreas is associated with pancreatic tissue fibrosis, and several previous studies have attempted to quantify pancreatic fibrosis and have suggested that a pancreas with less fibrosis, more fatty tissues, and more acinar cells is at risk for POPF [23]. Fujita et al. [23] reported a useful approach for quantifying pancreatic tissue objectively by acoustic radiation force impulse imaging, and pancreatic tissue fibrosis was found to be correlated with the overall incidence of POPF. In contrast, POPF after distal pancreatectomy is due to functional distal obstruction by the sphincter of Oddi complex at the ampulla [24]. Our study did not reveal the predictive factor of CR-POPF after distal pancreatectomy; further studies should be performed to evaluate CR-POPF after distal pancreatectomy.

In frail patients undergoing surgery, surgeons should consider various interventions preoperatively, intraoperatively, or postoperatively to reduce postoperative complications. Nutritional status and frailty are interrelated [25]; hence, preoperative intervention for nutritional status may improve frail status. In two randomized doubleblind studies [26, 27], both exercise and nutrition improved muscle mass, walking ability, and hematological parameters, possibly leading to the reversal of the frailty status. In these reports, resistance-type exercise training was effective in improving strength and physical performance in frail patients, and supplements were recommended during exercise training. This preoperative intervention is called "prehabilitation," which is a collective term to describe preoperative interventions aimed at increasing the physiological reserve of patients prior to surgery. Prehabilitation programs variably include physical, psychological, and nutritional interventions and may reduce the incidence of postoperative complications, shorten hospital stay, and improve health-related quality of life [28]. Despite the lack of evidence of improved mortality and duration of hospital stay, various beneficial prehabilitation programs for frail surgical 
Table 4 Univariate and multivariate analyses of predictive factors of postoperative complications with CD $\geqq$ IIla and CR-POPF in the pancreaticoduodenectomy group $(N=68)$

\begin{tabular}{|c|c|c|c|c|c|c|c|c|c|c|c|c|}
\hline \multirow[t]{3}{*}{ Factor } & \multicolumn{6}{|c|}{ Postoperative complications with CD $\geqq$ IIla } & \multicolumn{6}{|c|}{ CR-POPF } \\
\hline & \multicolumn{3}{|c|}{ Univariate } & \multicolumn{3}{|c|}{ Multivariate } & \multicolumn{3}{|c|}{ Univariate } & \multicolumn{3}{|c|}{ Multivariate } \\
\hline & $\begin{array}{l}p \\
\text { value }\end{array}$ & $\begin{array}{l}\text { Odds } \\
\text { ratio }\end{array}$ & $\begin{array}{l}95 \% \mathrm{Cl} \text { for } \\
\operatorname{Exp}(\mathrm{B})\end{array}$ & $\begin{array}{l}p \\
\text { value }\end{array}$ & $\begin{array}{l}\text { Odds } \\
\text { ratio }\end{array}$ & $\begin{array}{l}95 \% \mathrm{Cl} \text { for } \\
\operatorname{Exp}(\mathrm{B})\end{array}$ & $\begin{array}{l}p \\
\text { value }\end{array}$ & $\begin{array}{l}\text { Odds } \\
\text { ratio }\end{array}$ & $\begin{array}{l}95 \% \mathrm{Cl} \text { for } \\
\operatorname{Exp}(\mathrm{B})\end{array}$ & $\begin{array}{l}p \\
\text { value }\end{array}$ & $\begin{array}{l}\text { Odds } \\
\text { ratio }\end{array}$ & $\begin{array}{l}95 \% \mathrm{Cl} \text { for } \\
\operatorname{Exp}(\mathrm{B})\end{array}$ \\
\hline Age (years) & 0.62 & 1.036 & $0.986-1.089$ & & & & 0.339 & 1.031 & $0.968-1.099$ & & & \\
\hline Sex (female/male) & 0.669 & 1.240 & $0.463-3.323$ & & & & 0.313 & 0.543 & $0.166-1.779$ & & & \\
\hline BMI (kg/m2) & 0.771 & 0.997 & $0.984-1.011$ & & & & 0.331 & 1.007 & $0.993-1.022$ & & & \\
\hline Smoking & 0.672 & 1.230 & $0.442-3.210$ & & & & 0.818 & 0.871 & $0.266-2.848$ & & & \\
\hline Alcohol & 0.410 & 0.378 & $0.037-3.828$ & & & & 0.999 & 0.000 & 0.000 & & & \\
\hline Disease & 0.811 & 0.964 & $0.715-1.301$ & & & & 0.160 & 1.274 & $0.909-1.787$ & & & \\
\hline Diabetes mellitus & 0.209 & 2.114 & $0.657-6.801$ & & & & 0.437 & 0.526 & $0.104-2.661$ & & & \\
\hline $\begin{array}{l}\text { Antithrombotic } \\
\text { drugs }\end{array}$ & 0.210 & 2.041 & $0.669-6.225$ & & & & 0.729 & 1.262 & $0.338-4.707$ & & & \\
\hline $\begin{array}{l}\text { Operative time } \\
\text { (min) }\end{array}$ & 0.809 & 0.999 & $0.995-1.004$ & & & & 0.095 & 0.995 & 0.998-1.001 & 0.184 & 0.996 & $0.989-1.002$ \\
\hline Blood loss (g) & 0.980 & 1.000 & $0.999-1.001$ & & & & 0.554 & 1.000 & 0.999-1.001 & & & \\
\hline $\begin{array}{l}\text { Intraoperative } \\
\text { transfusion }\end{array}$ & 0.720 & 0.822 & $0.282-2.369$ & & & & 0.544 & 0.648 & $0.159-2.637$ & & & \\
\hline Soft pancreas & 0.074 & 2.471 & $0.916-6.666$ & 0.065 & 2.656 & $0.941-7.500$ & 0.021 & 6.462 & $1.319-31.663$ & 0.034 & 5.696 & $1.142-28.149$ \\
\hline Albumin (g/l) & 0.287 & 0.603 & $0.237-1.533$ & & & & 0.954 & 0.968 & $0.314-2.980$ & & & \\
\hline $\begin{array}{l}\text { Lymphocyte (x } \\
\left.10^{3} / \mu \mathrm{l}\right)\end{array}$ & 0.321 & 1.000 & $1.000-1.001$ & & & & 0.348 & 1.000 & $1.000-1.001$ & & & \\
\hline $\begin{array}{l}\text { Total cholesterol } \\
(\mathrm{mg} / \mathrm{dl})\end{array}$ & 0.138 & 0.991 & $0.979-1.003$ & & & & 0.148 & 0.988 & $0.972-1.004$ & & & \\
\hline Hemoglobin ( $\mathrm{g} / \mathrm{dl})$ & 0.533 & 1.046 & $0.907-1.207$ & & & & 0.701 & 0.968 & $0.821-1.141$ & & & \\
\hline PNI & 0.836 & 1.006 & $0.951-1.065$ & & & & 0.551 & 1.024 & $0.946-1.109$ & & & \\
\hline CONUT score & 0.483 & 0.779 & $0.388-1.565$ & & & & 0.162 & 0.504 & $0.193-1.317$ & & & \\
\hline Sarcopenia & 0.642 & 1.255 & $0.482-3.265$ & & & & 0.192 & 2.250 & $0.666-7.605$ & & & \\
\hline Frail & 0.054 & 5.104 & $0.975-26.713$ & 0.047 & 5.604 & $1.022-30.734$ & 0.896 & 1.119 & $0.266-6.092$ & & & \\
\hline
\end{tabular}

Abbreviations: $B M I$ body mass index, $C D$ Clavien-Dindo classification, COUNT controlling nutritional status, CR-POPF clinically relevant postoperative pancreatic fistula, $P N I$ prognostic nutritional index

patients have been reported in a systematic review [28]. Thus, we should consider both exercise and nutritional intervention preoperatively. Conversely, early postoperative nutritional support helps reduce the risk of postoperative complications, especially postoperative early enteral nutrition, which improves the nutritional status and promotes functional recovery of the digestive system [29]. As one of the intraoperative interventions, Gilliland et al. [30] recommended that in pancreatic cancer patients with moderately decreased albumin levels $(<3.0$ $\mathrm{mg} / \mathrm{dL}$ ) or weight loss $>5 \%$, jejunostomy feeding tubes should be used intraoperatively to avoid postoperative undesirable patient outcomes associated with insufficient nutritional intervention. Moreover, to avoid postoperative complications, it may be useful to insert an enteral tube after a more invasive surgery, such as pancreaticoduodenectomy, as an early nutritional support for frail patients with poor nutritional status.
In this study, three patients died; the main cause of death was acute respiratory failure. Postoperative complications (CD $\geq$ IIIa) in these three patients varied; two of them had CR-POPF. Considering our results, frail patients undergoing pancreaticoduodenectomy should have preoperative prehabilitation, especially respiratory prehabilitation [31]. In a study by Varga JT [31], a respiratory prehabilitation program provided a positive effect on the cardiovascular system, metabolism, muscles, and lung mechanics, resulting in optimal functional condition and less postoperative complication. This prehabilitation was supposed to improve nutritional status, strength, physical performance, and frail status. We need to consider the duration of prehabilitation as a long-duration prehabilitation program may result in disease progression, especially in pancreatic cancer or bile duct cancer patients. If the preoperative frail status does not improve, pancreatectomy should be avoided in frail patients and other 
Table 5 Univariate and multivariate analyses of predictive factors of postoperative complications with CD $\geqq$ IIla and CR-POPF in the distal pancreatectomy group $(N=25)$

\begin{tabular}{|c|c|c|c|c|c|c|c|c|c|c|c|c|}
\hline \multirow[t]{3}{*}{ Factor } & \multicolumn{6}{|c|}{ Postoperative complications with CD $\geqq$ IIla } & \multicolumn{6}{|c|}{ CR-POPF } \\
\hline & \multicolumn{3}{|c|}{ Univariate } & \multicolumn{3}{|c|}{ Multivariate } & \multicolumn{3}{|c|}{ Univariate } & \multicolumn{3}{|c|}{ Multivariate } \\
\hline & $\begin{array}{l}p \\
\text { value }\end{array}$ & $\begin{array}{l}\text { Odds } \\
\text { ratio }\end{array}$ & $\begin{array}{l}95 \% \mathrm{Cl} \text { for } \\
\operatorname{Exp}(\mathrm{B})\end{array}$ & $\begin{array}{l}p \\
\text { value }\end{array}$ & $\begin{array}{l}\text { Odds } \\
\text { ratio }\end{array}$ & $\begin{array}{l}95 \% \mathrm{Cl} \text { for } \\
\operatorname{Exp}(\mathrm{B})\end{array}$ & $\begin{array}{l}p \\
\text { value }\end{array}$ & $\begin{array}{l}\text { Odds } \\
\text { ratio }\end{array}$ & $\begin{array}{l}95 \% \mathrm{Cl} \text { for } \\
\operatorname{Exp}(\mathrm{B})\end{array}$ & $\begin{array}{l}p \\
\text { value }\end{array}$ & $\begin{array}{l}\text { Odds } \\
\text { ratio }\end{array}$ & $\begin{array}{l}95 \% \mathrm{Cl} \text { for } \\
\operatorname{Exp}(\mathrm{B})\end{array}$ \\
\hline Age (years) & 0.476 & 1.024 & $0.960-1.091$ & & & & 0.910 & 0.994 & $0.900-1.098$ & & & \\
\hline Sex (female/male) & 0.611 & 1.556 & $0.284-8.531$ & & & & 0.765 & 0.643 & $0.036-11.631$ & & & \\
\hline BMI (kg/m2) & 0.126 & 1.230 & $0.944-1.603$ & & & & 0.804 & 1.048 & $0.726-1.512$ & & & \\
\hline Smoking & 0.386 & 0.480 & $0.091-2.523$ & & & & 0.859 & 0.769 & $0.043-13.866$ & & & \\
\hline Alcohol & 0.999 & 0.000 & 0.000 & & & & 0.081 & 21.000 & $0.686-642.982$ & 0.997 & 0.000 & 0.000 \\
\hline Disease & 0.796 & 0.928 & $0.527-1.634$ & & & & 0.298 & 1.563 & 0.673-3.629 & & & \\
\hline Diabetes mellitus & 0.915 & 1.110 & $0.192-6.286$ & & & & 0.578 & 2.286 & $0.124-41.985$ & & & \\
\hline $\begin{array}{l}\text { Antithrombotic } \\
\text { drugs }\end{array}$ & 0.621 & 0.542 & $0.048-6.144$ & & & & 0.219 & 6.667 & $0.323-137.403$ & & & \\
\hline $\begin{array}{l}\text { Operative time } \\
\text { (min) }\end{array}$ & 0.868 & 1.001 & $0.992-1.010$ & & & & 0.865 & 1.001 & 0.986-1.017 & & & \\
\hline Blood loss (g) & 0.471 & 1.001 & $0.999-1.002$ & & & & 0.305 & 1.001 & $0.999-1.004$ & & & \\
\hline $\begin{array}{l}\text { Intraoperative } \\
\text { transfusion }\end{array}$ & 1.000 & 0.000 & 0.000 & & & & 1.000 & 0.000 & 0.000 & & & \\
\hline Soft pancreas & 1.000 & 0.000 & 0.000 & & & & 1.000 & 0.000 & 0.000 & & & \\
\hline Albumin (g/l) & 0.058 & 0.037 & $0.001-1.121$ & 0.366 & 0.108 & $0.001-13.492$ & 0.709 & 0.388 & $0.003-55.874$ & & & \\
\hline $\begin{array}{l}\text { Lymphocyte (x } \\
\left.10^{3} / \mu \mathrm{l}\right)\end{array}$ & 0.492 & 0.999 & $0.998-1.001$ & & & & 0.099 & 1.002 & $1.000-1.004$ & 0.997 & 1.020 & 0.000 \\
\hline $\begin{array}{l}\text { Total cholesterol } \\
\text { (mg/dl) }\end{array}$ & 0.602 & 1.006 & $0.983-1.031$ & & & & 0.097 & 0.970 & $0.936-1.006$ & 0.999 & 0.759 & 0.000 \\
\hline Hemoglobin (g/dl) & 0.697 & 0.887 & $0.484-1.625$ & & & & 0.993 & 0.995 & $0.344-2.881$ & & & \\
\hline PNI & 0.084 & 0.794 & $0.612-1.031$ & 0.559 & 0.901 & $0.634-1.279$ & 0.311 & 1.189 & $0.851-1.661$ & & & \\
\hline CONUT score & 0.511 & 1.760 & $0.326-9.510$ & & & & 0.671 & 1.875 & $0.103-34.131$ & & & \\
\hline Sarcopenia & 0.529 & 2.000 & $0.231-17.338$ & & & & 0.219 & 6.667 & $0.323-137.403$ & & & \\
\hline Frail & 0.671 & 1.875 & $0.103-34.131$ & & & & 0.077 & 22.000 & $0.719-672.782$ & 0.998 & 0.000 & 0.000 \\
\hline
\end{tabular}

Abbreviations: $B M I$ body mass index, $C D$ Clavien-Dindo classification, COUNT controlling nutritional status, CR-POPF clinically relevant postoperative pancreatic fistula, $P N I$ prognostic nutritional index

treatments, such as chemotherapy, radiotherapy, or chemoradiotherapy, should be considered. Surgery is a radical treatment, especially for pancreatic cancer and bile duct cancer. This problem is puzzling for many surgeons.

Consideration of frailty may be beneficial for the evaluation of operative risk and selection of patients.

This study has several limitations. First, this retrospective study as conducted on a very small scale compared with previous reports because of its singleinstitution setting; thus, future multi-institutional prospective research studies are needed. Second, although previous reports $[22,23]$ have revealed objective evaluation of pancreatic fibrosis preoperatively or postoperatively, soft pancreas was defined by surgeons subjectively in this study. In previous reports $[21,22]$ revealing a relationship between pancreatectomy and CR-POPF, surgeons had judged the pancreas as soft or hard subjectively. Third, the definition of frail varies $[16,20$, $32,33]$; thus, our result may be remarkably different than those of previous studies using other definitions. In our report, we adopted the J-CHS criteria, which was a simple frailty assessment tool, and included similar items to the criteria of sarcopenia. Finally, the timing of measuring physical activity and collection of blood samples were not planned and varied among patients. Furthermore, there were patients who underwent nutrition or exercise intervention after being diagnosed frail, and we did not evaluate the effectiveness after these interventions before pancreatectomy. Therefore, future prospective research studies are needed to confirm and evaluate these preliminary findings.

\section{Conclusion}

Frailty may be a useful predictive factor of postoperative complications in patients undergoing 
pancreaticoduodenectomy. Although many physicians instinctively and subjectively judge whether patients have the physiological reserve to endure operations and postoperative burdens, frailty might be a more effective risk stratification tool to identify potentially high-risk surgical patients undergoing pancreaticoduodenectomy.

\section{Abbreviations}

CD: Clavien-Dindo; Cl: Confidence interval; CR-POPF: Clinically relevant postoperative pancreatic fistula; J-CHS: Japanese version of the Cardiovascular Health Study; OR: Odds ratio; POPF: Postoperative pancreatic fistula

\section{Acknowledgements}

We would like to thank Editage (www.editage.jp) for their English language editing services and express our special thanks to Ayano Takeuchi, who is a statistician of Keio University School of Medicine, for checking our statistical analysis.

\section{Authors' contributions}

All authors helped to perform the research; NY manuscript writing and performing data analysis; YH, ST, YT, MK, and NS performing data analysis; $\mathrm{NR}$, TH, and ET performing data analysis and drafting conception and design. All authors read and approved the final manuscript.

\section{Funding}

There is no funding for this work.

\section{Availability of data and materials}

The datasets used and analyzed during the current study are available from the corresponding author on reasonable request

\section{Ethics approval and consent to participate}

We conducted a retrospective observational study and used the "opt-out" method as a way to obtain informed content from patients. The study was approved by the Human Experimentation Committee of our institution (ethical approval number: 20190032)

\section{Consent for publication}

We have obtained the consent for publication from all patients.

\section{Competing interests}

The authors declare that they have no competing interests.

\section{Author details}

'Department of Surgery, Saiseikai Yokohamashi Tobu Hospital, 3-6-1 Shimosueyoshi, Tsurumi-ku, Yokohama, Kanagawa 230-0012, Japan.

${ }^{2}$ Department of Patients Support Center, Saiseikai Yokohamashi Tobu Hospital, 3-6-1 Shimosueyoshi, Tsurumi-ku, Yokohama, Kanagawa 230-0012, Japan.

Received: 9 May 2020 Accepted: 24 July 2020

Published online: 03 August 2020

\section{References}

1. Fried LP, Tangen CM, Walston J, Newman AB, Hirsch C, Gottdiener J, et al. Frailty in older adults: evidence for a phenotype. J Gerontol A Biol Sci Med Sci. 2001;56(3):146-56.

2. Ragulin-Coyne E, Carroll JE, Smith JK, Witkowski ER, Ng SC, Shah SA, et al. Perioperative mortality after pancreatectomy: a risk score to aid decisionmaking. Surgery. 2012;152(3):120-7.

3. Pope D, Ramesh H, Gennari R, Corsini G, Maffezzini M, Hoekstra HJ, et al. Pre-operative assessment of cancer in the elderly (PACE): a comprehensive assessment of underlying characteristics of elderly cancer patients prior to elective surgery. Surg Oncol. 2006;15(4):189-97.

4. Lee DY, Schwartz JA, Wexelman B, Kirchoff D, Yang KC, Attiyeh F. Outcomes of pancreaticoduodenectomy for pancreatic malignancy in octogenarians: an American college of surgeons national surgical quality improvement program analysis. Am J Surg. 2014;207(4):540-8.
5. Augustin T, Burstein MD, Schneider EB, Morris-Stiff G, Wey J, Chalikonda S, et al. Frailty predicts risk of life-threatening complications and mortality after pancreatic resections. Surgery. 2016;160(4):987-96.

6. Sabesan A, Gough BL, Anderson C, Abdel-Misih R, Petrelli NJ, Bennett Jر. High volume pancreaticoduodenectomy performed at an academic community cancer center. Am J Surg. 2019;218(2):349-54.

7. Cesari M, Landi F, Vellas B, Bernabei R, Marzetti E. Sarcopenia and physical frailty: two sides of the same coin. Front Aging Neurosci. 2014;6:192.

8. Valero V 3rd, Amini N, Spolverato G, Weiss MJ, Hirose K, Dagher NN, et al. Sarcopenia adversely impacts postoperative complications following resection or transplantation in patients with primary liver tumors. J Gastrointest Surg. 2015;19(2):272-81.

9. Ratnayake CB, Loveday BP, Shrikhande SV, Windsor JA, Pandanaboyana S. Impact of preoperative sarcopenia on postoperative outcomes following pancreatic resection: a systematic review and meta-analysis. Pancreatology. 2018;18(8):996-1004

10. Mogal H, Vermilion SA, Dodson R, Hsu FC, Howerton R, Shen P, et al. Modified frailty index predicts morbidity and mortality after pancreaticoduodenectomy. Ann Surg Oncol. 2017;24(6):1714-21.

11. Guyton RL Jr, Mosquera C, Spaniolas K, Fitzgerald TL. Association of increasing frailty with detrimental outcomes after pancreatic resection. Am Surg. 2018;84(4):512-9.

12. Taniguchi H, Sasaki T, Fujita H, Kobayashi H, Kawasaki R, Ogata T, et al. Effects of goal-directed fluid therapy on enhanced postoperative recovery: an interventional comparative observational study with a historical control group on oesophagectomy combined with ERAS program. Clin Nutr ESPEN. 2018;23:184-93.

13. Garcea G, Ladwa N, Neal CP, Metcalfe MS, Dennison AR, Berry DP. Preoperative neutrophil-to-lymphocyte ratio $(\mathrm{n} / \mathrm{r})$ is associated with reduced disease-free survival following curative resection of pancreatic adenocarcinoma. World J Surg. 2011;35(4):868-72.

14. Kato $Y$, Yamada S, Suenaga $M$, Takami H, Niwa $Y$, Hayashi $M$, et al. Impact of the controlling nutritional status score on the prognosis after curative resection of pancreatic ductal adenocarcinoma. Pancreas. 2018:47(7):823-9.

15. Chen LK, Liu LK, Woo J, Assantachai P, Auyeung TW, Bahyah KS, et al. Sarcopenia in Asia: consensus report of the Asian working Group for Sarcopenia. J Am Med Dir Assoc. 2014;15(2):95-101.

16. Satake S, Shimada H, Yamada M, Kim H, Yoshida H, Gondo Y, et al. Prevalence of frailty among community-dwellers and outpatients in Japan as defined by the Japanese version of the Cardiovascular Health Study criteria. Geriatr Gerontol Int. 2017;17(12):2629-34.

17. Bassi C, Marchegiani G, Dervenis C, Sarr M, Abu Hilal M, Adham M, et al. The 2016 update of the International Study Group (ISGPS) definition and grading of postoperative pancreatic fistula: 11 years after. Surgery. 2017; 161(3):584-91.

18. Martin AN, Narayanan S, Turrentine FE, Bauer TW, Adams RB, Zaydfudim VM Pancreatic duct size and gland texture are associated with pancreatic fistula after pancreaticoduodenectomy but not after distal pancreatectomy. PLoS One. 2018;13(9):e0203841.

19. Revenig LM, Canter DJ, Taylor MD, Tai C, Sweeney JF, Sarmiento JM, et al. Too frail for surgery? initial results of a large multidisciplinary prospective study examining preoperative variables predictive of poor surgical outcomes. J Am Coll Surg. 2013:217(4):665-70.

20. Velanovich V, Antoine H, Swartz A, Peters D, Rubinfeld I. Accumulating deficits model of frailty and postoperative mortality and morbidity: its application to a national database. J Surg Res. 2013;183(1):104-10.

21. Wellner UF, Kayser G, Lapshyn H, Sick O, Makowiec F, Höppner J, et al. A simple scoring system based on clinical factors related to pancreatic texture predicts postoperative pancreatic fistula preoperatively. HPB (Oxford). 2010; 12(10):696-702

22. Pedrazzoli S. Pancreatoduodenectomy (PD) and postoperative pancreatic fistula (POPF): a systematic review and analysis of the POPF-related mortality rate in 60,739 patients retrieved from the English literature published between 1990 and 2015. Medicine (Baltimore). 2017:96(19):e6858.

23. Fujita Y, Kitago M, Abe T, Itano O, Shinoda M, Abe Y, et al. Evaluation of pancreatic fibrosis with acoustic radiation force impulse imaging and automated quantification of pancreatic tissue components. Pancreas. 2018; 47(10):1277-82

24. Hackert T, Klaiber U, Hinz U, Kehayova T, Probst P, Knebel P, et al. Sphincter of Oddi botulinum toxin injection to prevent pancreatic fistula after distal pancreatectomy. Surgery. 2017 May;161(5):1444-50. 
25. Boulos C, Salameh P, Barberger-Gateau P. Malnutrition and frailty in community dwelling older adults living in a rural setting. Clin Nutr. 2016; 35(1):138-43.

26. Tieland M, Dirks ML, van der Zwaluw N, Verdijk LB, van de Rest $\mathrm{O}$, de Groot LC, et al. Protein supplementation increases muscle mass gain during prolonged resistance-type exercise training in frail elderly people: a randomized, double-blind, placebo-controlled trial. J Am Med Dir Assoc. 2012;13(8):713-9.

27. Kim H, Suzuki T, Kim M, Kojima N, Ota N, Shimotoyodome A, et al. Effects of exercise and milk fat globule membrane (MFGM) supplementation on body composition, physical function, and hematological parameters in community-dwelling frail Japanese women: a randomized double blind, placebo-controlled, follow-up trial. PLoS One. 2015;10(2):e0116256.

28. Borrell-Vega J, Esparza Gutierrez AG, Humeidan ML. Multimodal prehabilitation programs for older surgical patients. Anesthesiol Clin. 2019: 37(3):437-52.

29. Shu XL, Kang K, Gu $\sqcup$, Zhang YS. Effect of early enteral nutrition on patients with digestive tract surgery: a meta-analysis of randomized controlled trials. Exp Ther Med. 2016;12(4):2136-44.

30. Gilliland TM, Villafane-Ferriol N, Shah KP, Shah RM, Tran Cao HS, Massarweh $\mathrm{NN}$, et al. Nutritional and metabolic derangements in pancreatic cancer and pancreatic resection. Nutrients. 2017;9(3): pii: E243.

31. Varga JT. Smoking and pulmonary complications: respiratory prehabilitation. J Thorac Dis. 2019;11:S639-44.

32. Gobbens RJ, Schols JM, van Assen MA. Exploring the efficacy of Tilburg frailty indicator: a review. Clin Interv Aging. 2017:12:1739-52.

33. Sewo Sampaio PY, Sampaio RA, Yamada M, Arai H. Systematic review of the Kihon Checklist: is it a reliable assessment of frailty? Geriatr Gerontol Int. 2016;16(8):893-902.

\section{Publisher's Note}

Springer Nature remains neutral with regard to jurisdictional claims in published maps and institutional affiliations.

Ready to submit your research? Choose BMC and benefit from:

- fast, convenient online submission

- thorough peer review by experienced researchers in your field

- rapid publication on acceptance

- support for research data, including large and complex data types

- gold Open Access which fosters wider collaboration and increased citations

- maximum visibility for your research: over $100 \mathrm{M}$ website views per year

At $\mathrm{BMC}$, research is always in progress.

Learn more biomedcentral.com/submissions 